\title{
Lupus Miliaris Disseminatus Faciei versus Granulomatous Rosacea: A Case Report
}

\author{
Ji-In Seo Min Kyung Shin \\ Department of Dermatology, School of Medicine, Kyung Hee University, Seoul, Republic of \\ Korea
}

\section{Keywords}

Lupus miliaris disseminatus faciei · Granulomatous rosacea - Acne agminata - Lupoid rosacea

\begin{abstract}
Lupus miliaris disseminatus faciei (LMDF) and granulomatous rosacea are 2 distinct inflammatory dermatoses with overlapping clinical features: reddish-yellow papular eruptions localized on the central face. Consequently, LMDF can easily be misdiagnosed as granulomatous rosacea or vice versa. Because delayed treatment in LMDF may increase chances of permanent scar formation, accurate diagnosis is important. We therefore analyzed published literature and case studies to organize the essential features differentiating LMDF from granulomatous rosacea. In addition, we report each case of LMDF and granulomatous rosacea for direct comparison.
\end{abstract}

\section{Introduction}

Lupus miliaris disseminatus faciei (LMDF) is an inflammatory dermatosis characterized by "disseminated" reddish-yellow papular eruptions. The central face - especially the periorbital and perioral area - is primarily involved, but extra-facial manifestations are not uncommon [1]. The term "lupus" originates from LMDF being classified as tuberculids based on its histopathological hallmark: dermal epithelioid granulomas with caseation necrosis. Yet, repetitive failure to detect Mycobacterium tuberculosis from the lesions has isolated LMDF from cutaneous tuberculosis [2, 3]. Instead, clinical features resembling clustered synonym for "agminis" - acne lesions around the eyelids and lips presented "acne agminata" as a widely accepted terminology [4]. In the 1980s, however, overlapping features with rosacea grouped LMDF as a specialized variant of rosacea: granulomatous or lipoid rosacea 
[5]. Nowadays, due to phenotypical and histopathological differences, LMDF is considered an unique entity with "rosacea-like" features [6]. Autoimmune reaction toward pilosebaceous units triggered by hair follicle damage or epidermal cyst rupture is the currently proposed etiopathogenesis [5, 7]. To highlight LMDF as a distinct entity separated from similar granulomatous dermatoses, scientists have proposed a new nomenclature: FIGURE standing for "facial idiopathic granulomas with regressive evolution" [8].

In clinical practice, however, LMDF can easily be misdiagnosed as granulomatous rosacea and vice versa. The classical LMDF phenotype - yellow to red firm papules on the center face - is also a common manifestation of granulomatous rosacea. Furthermore, the broad definition of rosacea obscures its distinction with LMDF. Based on the 2002 standard classification of rosacea by the National Rosacea Society [9], granulomatous rosacea is a variant which manifests with noninflammatory uniform papules on the central face. Interestingly, other conventional symptoms of rosacea - flushing, nontransient erythema, telangiectasia, and stinging sensation - are not necessary for diagnosis. In the updated 2017 standard classification [10], diagnostic phenotype (fixed centrofacial erythema) or 2 major phenotypes (flushing, papules and pustules, telangiectasia and ocular manifestations) are required for confirmation. As demonstrated, diagnosis of rosacea solely depends on its clinical features, and the overlapping phenotypes with LMDF may confuse judgment.

Despite diagnostic challenge, meticulous clinical examination, history taking, histopathologic evaluation, and treatment response follow-ups can guide clinicians toward accurate diagnosis. We therefore analyzed published literature and case studies to organize the essential features differentiating LMDF from granulomatous rosacea. In addition, we report each case of LMDF and granulomatous rosacea for direct comparison.

\section{Case 1}

The first case is a 62-year-old man who visited our dermatology clinic for asymptomatic papules on the face. The lesions appeared 2 months ago and did not respond to general treatments in the primary care hospitals. Physical examination showed dense erythematous papules localized on the central face, involving the upper and lower eyelids; background erythema was absent (Fig. 1a). Initially, the patient was diagnosed with granulomatous rosacea and treated with systemic minocycline $100 \mathrm{mg} /$ day and topical pimecrolimus. Despite 2 weeks of treatment, symptoms showed no improvement and worsened. Under the possibility of LMDF, systemic steroid (deflazacort) $12 \mathrm{mg} /$ day was added. To further exclude other granulomatous dermatoses, skin biopsy and chest X-ray were performed. Biopsy from the cheek displayed epithelioid cell granulomas with central caseous necrosis in the dermis (Fig. 1c, d); the acid-fast bacilli (AFB) stain was negative. The chest X-ray showed no active lesions. Systemic steroid treatment improved lesions within 2 weeks (Fig. 1b), and the patient continued the treatment for 14 weeks until symptoms subsided. A month after discontinuing steroid treatment, however, erythematous papules reappeared on the same locations. Systemic steroid (deflazacort) $12 \mathrm{mg} /$ day was reapplied along with roxithromycin $150 \mathrm{mg} /$ day. Once again, lesions receded and the patient is currently tapering the steroid dosage in his follow-ups.

\section{Case 2}

Our second case involves a 61-year-old female who visited the dermatology clinic for papules on the eyelids and perioral area, accompanied by transient facial flushing. The lesions appeared 3 months ago and did not respond to topical treatments in the primary care hospital.

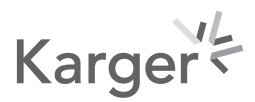



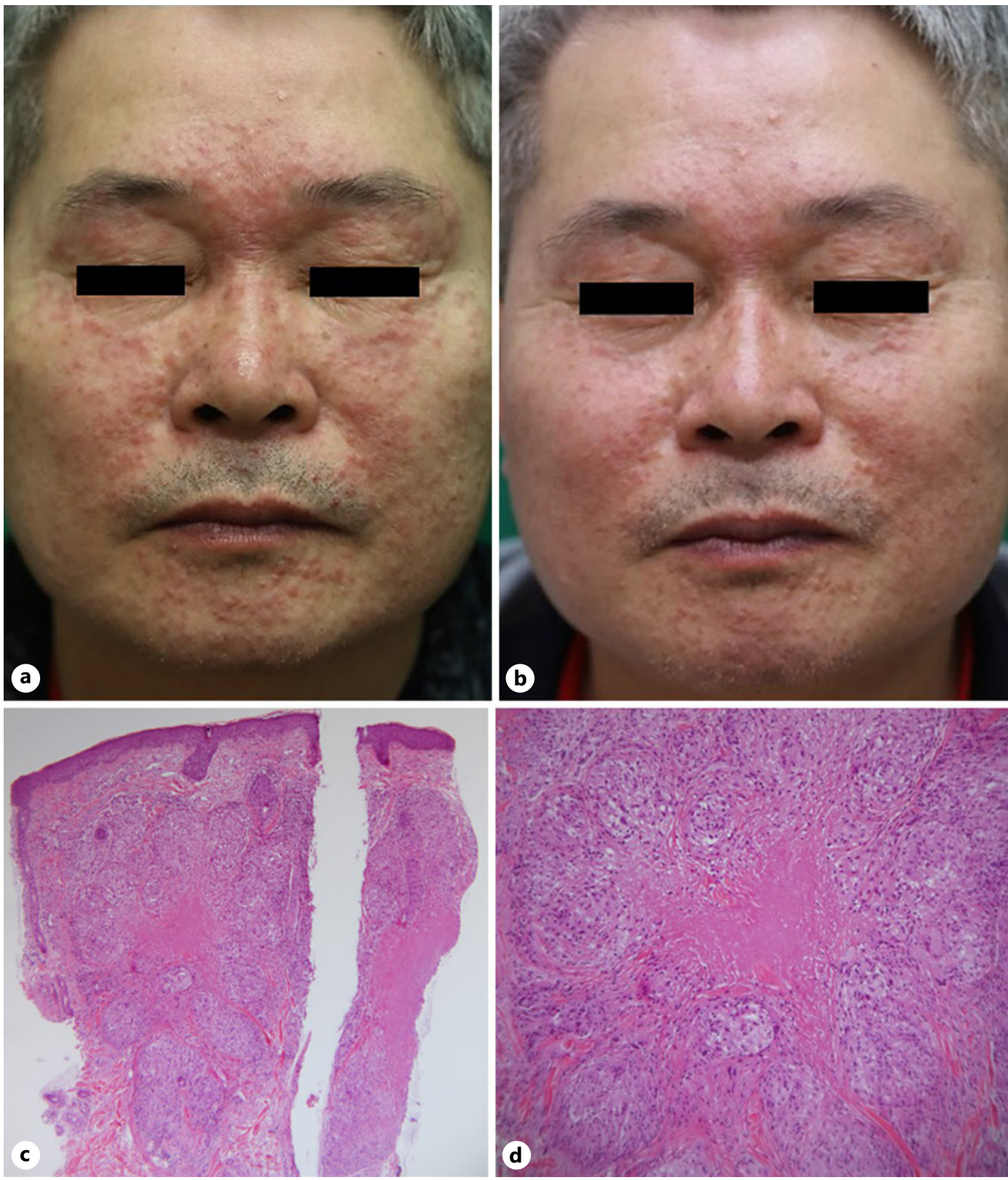

Fig. 1. a Multiple erythematous papules on the central face, involving the upper and lower eyelids; Background erythema is absent. b Improving lesions after 2 weeks of systemic steroid therapy. c Histopathologic finding showing multiple granulomatous infiltration in the upper dermis with central necrosis. HE. $\times 40$. d Epithelioid granulomas with central caseating necrosis. HE. $\times 100$.

Physical examination displayed erythematous papules localized on both eyelids and perioral area (Fig. 2a, b). The patient was diagnosed with granulomatous rosacea and started systemic minocycline therapy at a dosage of $100 \mathrm{mg} /$ day. Meanwhile, serum angiotensin-converting enzyme, serum interferon-gamma release assay, and chest X-ray were examined to exclude other granulomatous dermatosis; no abnormalities were detected. Skin biopsy displayed noncaseating epithelioid granulomas in the dermis (Fig. 3a, b) with marked solar elastosis and vessel dilatation (Fig. 3c); the AFB stain was negative. Although lesions showed partial improvement within 2 weeks, the oral medication was discontinued due to indigestion 

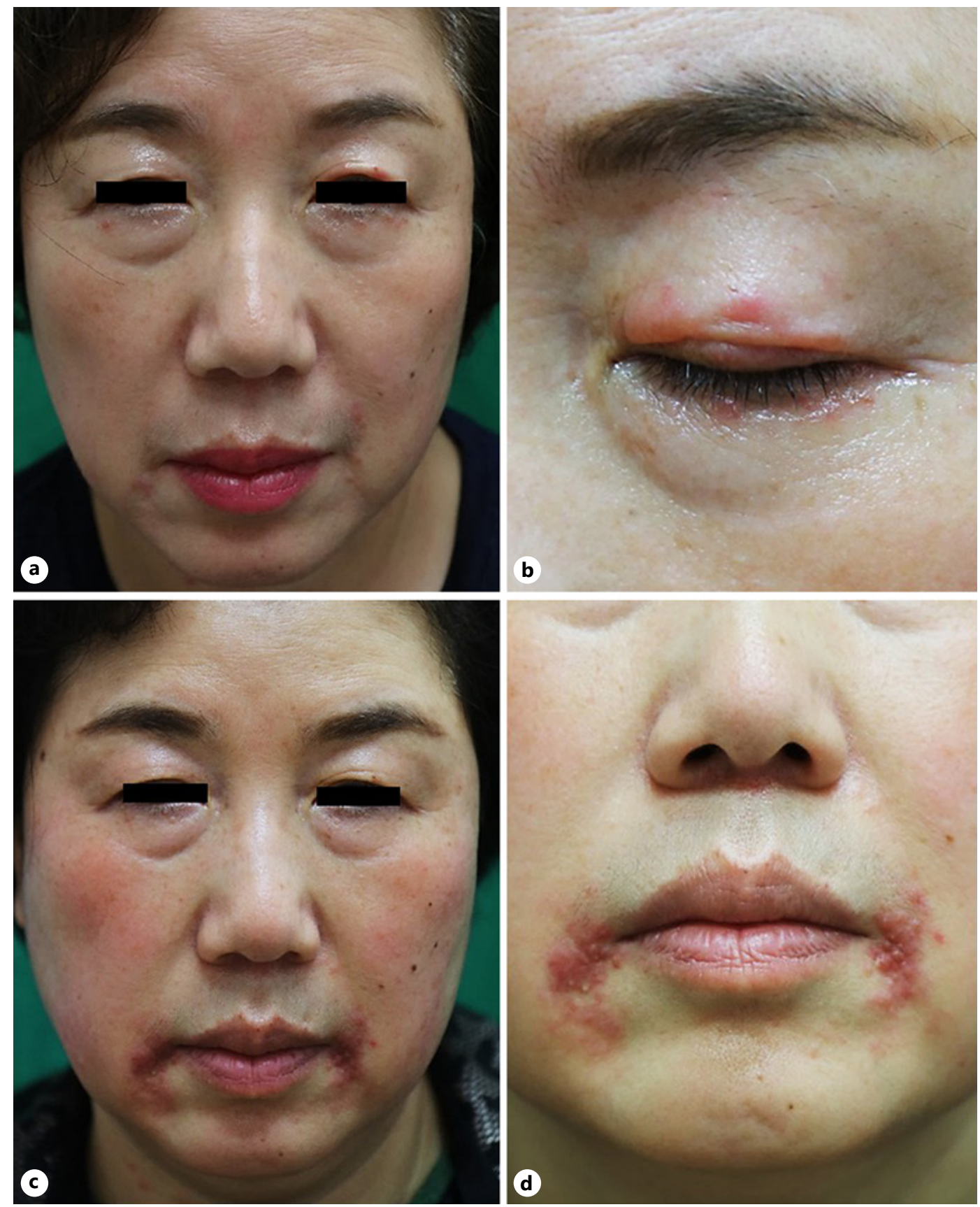

Fig. 2. a Erythematous papules localized on both eyelids and the perioral area. $\mathbf{b}$ The upper and lower eyelids are all involved. c After 12 weeks of treatment, eyelid lesions have cleared but perioral lesions aggravated. Her flushing also worsened, forming fixed erythema on both cheeks. d Grouped erythematous papules are symmetrically located on the perioral area.

problems. Instead, topical tacrolimus (perioral) and topical steroid (eyelid) were continued for 12 weeks. Despite improvements in the eyelid lesions, the perioral lesions aggravated and formed symmetric grouped erythematous papules (Fig. 2c, d). Her flushing also worsened and progressed into fixed erythema on both cheeks (Fig. 2c). Systemic doxycycline $100 \mathrm{mg} /$ day and systemic $\beta$-blocker (carvedilol) $3 \mathrm{mg} /$ day were added for 3 weeks but showed no improvement. Medications were changed to systemic steroid (methylprednisolone) $4 \mathrm{mg} /$ 


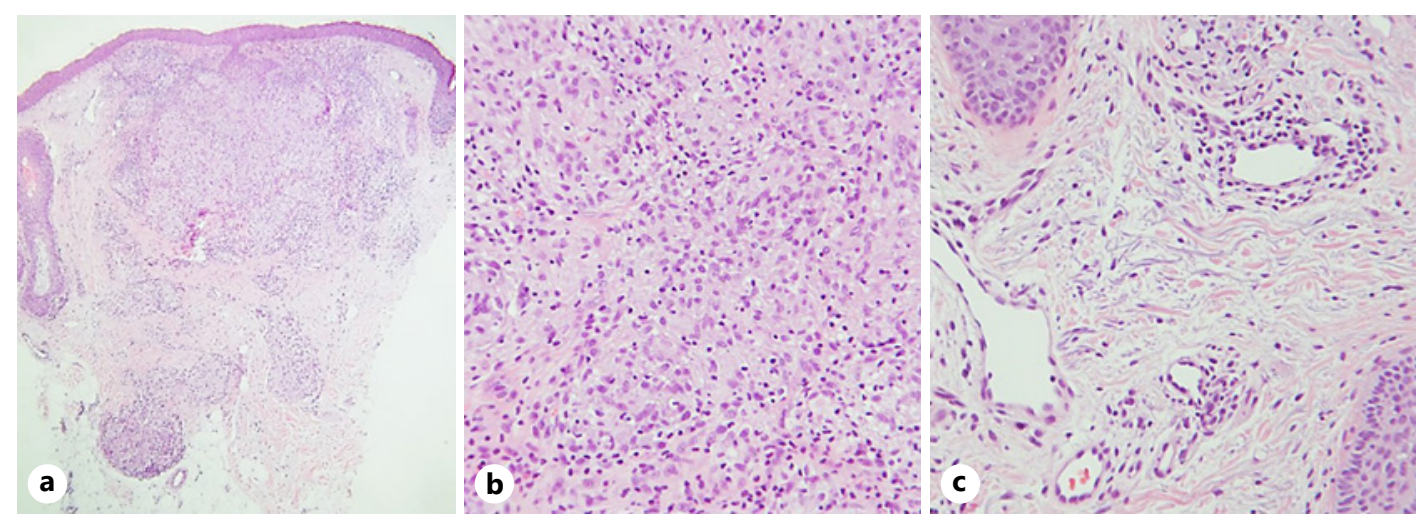

Fig. 3. a Histopathologic finding showing non-caseating granulomatous infiltration in the upper dermis. HE. $\times 40$. $\mathbf{b}$ The granuloma is composed of epithelioid cells and absent of central necrosis. HE. $\times 200$. c Marked solar elastosis with vessel dilatation in the upper dermis. HE. $\times 200$.

day and systemic roxithromycin $150 \mathrm{mg} /$ day. The lesions lessened in 3 weeks, and the patient continued the treatment for 4 additional weeks until lesions diminished. Since then, the patient has not experienced any relapse in 8 months.

\section{Discussion}

The 2 distinct disease entities - LMDF and granulomatous rosacea - share common clinical features. Our case 1 patient exemplifies how misdiagnosis can be easily made, especially during the patient's initial hospital visits when clinicians solely depend on clinical symptoms for diagnosis. Nevertheless, there are several valuable clinical, symptomatic, histologic, therapeutic, and prognostic features which distinguish LMDF from granulomatous rosacea. We have organized the key differences in Table 1 . In addition, direct comparison of patient 1 and 2 is summarized in Table 2.

LMDF lesions commonly appear on the eyelids, lack an erythematous base, and may spread to extra-facial locations. While granulomatous rosacea usually spares the eyelids and periorbital area, eyelid involvement is prevalent in LMDF and may be the sole manifestation [11-13]. In addition, fixed background erythema and irritating skin symptoms - burning, stinging, or dryness - are common in rosacea whereas less in LMDF $[1,3,6]$. Such symptoms aggravate due to external exposures (alcohol, sunlight, extremes of temperature, etc.) in granulomatous rosacea, while LMDF patients remain asymptomatic. Lastly, rosacea only involves the face and eyes, whereas LMDF may appear on extra-facial sites such as the axillae, neck, scalp, legs, trunk, and genitalia [1].

In regards to histopathology, dermal epithelioid granuloma with caseous necrosis is the hallmark of LMDF. Both entities present with epithelioid cell granulomas on the dermis, centered on pilosebaceous units $[5,11]$. Caseous necrosis, however, is absent in granulomatous rosacea [3]. Nonetheless, early stages of LMDF may only present as sarcoidal granulomas with peri-adnexal lymphocyte infiltrate, and late stages as dense dermal fibrosis [14]. In such cases, features favoring granulomatous rosacea - presence of dense lymphoid infiltrate, Demodex folliculorum infestation, solar elastosis, and capillary dilatation - should be searched for differential diagnosis [1, 3].

For treatment, LMDF shows consistent treatment response to systemic steroids [11, 15], while granulomatous rosacea to systemic tetracyclines. Likewise, LMDF patients show 
Table 1. Comparison of LMDF and granulomatous rosacea

\begin{tabular}{|c|c|c|}
\hline & LMDF & Granulomatous rosacea \\
\hline Synonyms & $\begin{array}{l}\text { Acne agminated, } \\
\text { Facial idiopathic granulomas with } \\
\text { regressive evolution (FIGURE) }\end{array}$ & Lupoid Rosacea \\
\hline \multirow[t]{4}{*}{$\begin{array}{l}\text { Clinical } \\
\text { manifestation }\end{array}$} & \multicolumn{2}{|c|}{ Discrete, flesh-colored, or mildly erythematous dome-shaped papules } \\
\hline & $\begin{array}{l}\text { Extra-facial lesions } \\
\text { Axillae, neck, scalp, legs, trunk, genitalia }\end{array}$ & Limited to face \\
\hline & $\begin{array}{l}\text { Eyelids (especially lower eyelid) } \\
\text { Upper lip }\end{array}$ & Eyelids usually spared \\
\hline & Absent & $\begin{array}{l}\text { Erythematosus base with } \\
\text { telangiectasia }\end{array}$ \\
\hline \multirow[t]{6}{*}{ Histopathology } & \multicolumn{2}{|c|}{ Epithelioid cell granulomas centered around pilosebaceous units } \\
\hline & Central necrosis & No necrosis \\
\hline & Not common & Significant lymphoid infiltrate \\
\hline & Not common & Presence of Demodex folliculorum \\
\hline & Not common & Capillary dilatation \\
\hline & Not common & Solar elastosis \\
\hline Gender & Male & Female \\
\hline Symptom & Asymptomatic & Flushing, itching, burning \\
\hline $\begin{array}{l}\text { Aggravation } \\
\text { factors }\end{array}$ & None & $\begin{array}{l}\text { Hot drinks, alcohol, sunlight, } \\
\text { extremes of temperature }\end{array}$ \\
\hline Respond to tetracycline & Inconsistent & Consistent \\
\hline Response to steroid & Consistent & May aggravate \\
\hline Scarring & Generally present & Absent \\
\hline Prognosis & $\begin{array}{l}\text { Spontaneous resolution } \\
\text { (12-24 months) }\end{array}$ & Chronic \\
\hline
\end{tabular}

LMDF, Lupus miliaris disseminatus faciei.

inconsistent responses to systemic tetracyclines [11, 12], and granulomatous rosacea may aggravate by systemic steroids. Although systemic tetracyclines are currently the first-line treatment recommended for LMDF, efficacy varies among patients [16]. On the contrary, low-dose systemic steroids not only achieve lesion improvement, but also prevent permanent scarring and new lesion occurrence $[11,13]$. Prognosis also shows opposing trends; LMDF shows spontaneous resolution - usually within 12-24 months - whereas granulomatous rosacea manifests a chronic course [12]. Despite self-resolution, LMDF generally leaves behind scarring while granulomatous rosacea does not. Therefore, early diagnosis and treatment is crucial for prevention of scars in LMDF.

Last but not least, excluding other cutaneous granulomatous disorders is important. Lupus vulgaris shows nearly identical histology to LMDF but demonstrates positivity in serum interferon-gamma release assay and skin biopsy AFB staining. When tuberculids progress, erythematous papules grow into nodules and ulcerate. On the other hand, histology of sarcoidosis resembles that of granulomatous rosacea. In such cases, blood test for increased angiotensin-converting enzyme levels, chest X-ray for bilateral hilar lymphadenopathy, and history taking for systemic symptoms (respiratory, gastrointestinal, etc.) should be checked 
Table 2. Comparison of patient 1 and 2

\begin{tabular}{lll}
\hline & Patient 1 (LMDF) & Patient 2 (Granulomatous rosacea) \\
\hline Clinical manifestation & Discrete, flesh-colored, or mildly erythematous dome-shaped papules \\
& Central face & \\
& Prominent eyelid involvement & Less eyelid involvement \\
& Upper lip involvement & Later eyelid spared \\
& Absent & Upper lip spared \\
Histopathology & Epithelioid cell granulomas on the dermis \\
& Central necrosis & Erythematosus base \\
& Less & Absent \\
& Less & Lymphoid infiltrate \\
Lender & Less & Solar elastosis \\
Symptom & Male & Capillary dilatation \\
Respond to tetracycline & Asymptomatic & Female \\
Response to steroid & No & Flushing \\
Scarring & Present & Yes \\
Prognosis & Resolved within 15 months & Yes \\
\hline LMDF, Lupus miliaris disseminatus faciei. & Absent \\
\hline & & Currently resolved state \\
\hline
\end{tabular}

for sarcoidosis. Additionally, the "apple jelly" colored red-brown lesions - rather than bright erythematous papules - characterize cutaneous sarcoidosis. The histology of granulomatous periorificial dermatitis (GPD), also named facial Afro-Caribbean childhood eruption, is also indistinguishable to that of granulomatous rosacea. Clinical features of GPD highly resemble our case patient 2: grouped firm papules on the perioral area (Fig. 2c, d). Nonetheless, GPD exclusively involves pre-pubertal children and is therefore suggested to be a childhood variant of granulomatous rosacea [17]. Likewise, perioral dermatitis presents with erythematous papules and pustules around the mouth. Fortunately, skin biopsy clearly differentiates perioral dermatitis with granulomatous rosacea: nonspecific perifollicular inflammatory infiltration is prevalent, and epithelioid granuloma is absent in perioral dermatitis.

The various nomenclature used to describe LMDF and granulomatous rosacea signifies the ambiguity of both dermatoses. Despite on-going controversies whether the 2 granulomatous disorders arise from a single origin, our analysis demonstrates apparent differences in their phenotype and pathology. Appliance of Table 1 in our case patients would have clarified the diagnosis process and enabled early diagnosis. Patient 1 , for instance, could have been diagnosed with LMDF on his first visit by the presence of dense erythematous papules on the eyelids, absence of background erythema or telangiectasia, and lack of sensitive skin symptoms. Fast improvements with systemic steroids would have strengthened the initial diagnosis. Skin biopsy presenting with epithelioid granulomas and central necrosis would have confirmed the diagnosis. As to patient 2, although lesions were localized on her eyelids, early diagnosis was possible due to her flushing symptoms and histopathologic findings: marked solar elastosis, dilated upper dermis vessels, and noncaseating granuloma. Future dermatologists may encounter similar situations in clinical practice. We believe our paper's organized comparison between LMDF and granulomatous rosacea will aid clinicians to accurately diagnose both dermatoses and promptly provide therapeutic interventions.

\section{Karger'}




\section{Statement of Ethics}

The research was conducted ethically in accordance with the World Medical Association Declaration of Helsinki. Written informed consent was taken from the 2 patients for the publication of case and clinical images. The paper is exempt from Ethical Committee approval since we present a case report that does not include an experimental or observational study.

\section{Conflict of Interest Statement}

The authors have no conflicts of interest to declare.

\section{Funding Sources}

This research did not receive any specific grant from the public, commercial, or not-forprofit sector.

\section{Author Contributions}

J.I.S. constructed the idea, performed literature review, and wrote the manuscript. M.K.S. reviewed the manuscript, provided critical feedback, and supervised the overall paper.

\section{References}

1 Sehgal VN, Srivastava G, Aggarwal AK, Belum VR, Reddy V, Sharma S. Lupus miliaris disseminatus faciei part II: an overview. Skinmed. 2005;4(4):234-8.

2 Hodak E, Trattner A, Feuerman H, Feinmesser M, Tsvieli R, Mitrani-Rosenbaum S, et al. Lupus miliaris disseminatus faciei: the DNA of Mycobacterium tuberculosis is not detectable in active lesions by polymerase chain reaction. Br J Dermatol. 1997;137(4):614-9.

3 Chougule A, Chatterjee D, Yadav R, Sethi S, De D, Saikia UN. Granulomatous rosacea versus lupus miliaris disseminatus faciei-2 faces of facial granulomatous disorder: a clinicohistological and molecular study. Am J Dermatopathol. 2018;40(11):819-23.

4 Dawson GW. Acne Agminata. Proc R Soc Med. 1909;2(Dermatol Sect):45-8.

5 Shitara A. Clinicopathological and immunological studies of lupus miliaris disseminatus faciei. J Dermatol. 1982;9(5):383-95.

6 van de Scheur MR, van der Waal RI, Starink TM. Lupus miliaris disseminatus faciei: a distinctive rosacea-like syndrome and not a granulomatous form of rosacea. Dermatology. 2003;206(2):120-3.

7 Makkar R, Ramesh V. On the diagnosis of facial granulomatous dermatoses of obscure origin. Int J Dermatol. 2005;44(7):606-9.

8 Skowron F, Causeret AS, Pabion C, Viallard AM, Balme B, Thomas L, et al. facial idiopathic granulomas with regressive evolution. is "lupus miliaris disseminatus faciei" still an acceptable diagnosis in the third millennium? Dermatology. 2000;201(4):287-9.

9 Wilkin J, Dahl M, Detmar M, Drake L, Feinstein A, Odom R, et al. Standard classification of rosacea: report of the National Rosacea Society Expert Committee on the Classification and Staging of Rosacea. J Am Acad Dermatol. 2002;46(4):584-7.

10 Gallo RL, Granstein RD, Kang S, Mannis M, Steinhoff M, Tan J, et al. Standard classification and pathophysiology of rosacea: the 2017 update by the National Rosacea Society Expert Committee. J Am Acad Dermatol. 2018; 78(1):148-55.

11 Al-Mutairi N. Nosology and therapeutic options for lupus miliaris disseminatus faciei. J Dermatol. 2011;38(9): 864-73.

12 Amiruddin D, Mii S, Fujimura T, Katsuoka K. Clinical evaluation of 35 cases of lupus miliaris disseminatus faciei. J Dermatol. 2011;38(6):618-20.

13 O’Driscoll T, Morgan G. Acne agminata of the eyelid. Proc R Soc Med. 1974;67(9):869-70.

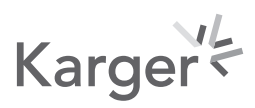


14 El Darouti M, Zaher H. Lupus miliaris disseminatus faciei: pathologic study of early, fully developed, and late lesions. Int J Dermatol. 1993;32(7):508-11.

15 Matsubara T. Clinical experiment on lupus vulgaris and lupus miliaris disseminatus faciei with single administration of corticoids. J Invest Dermatol. 1962;38:25-9.

16 Uesugi Y, Aiba S, Usuba M, Tagami H. Oral prednisone in the treatment of acne agminata. Br J Dermatol. 1996; 134(6):1098-100.

17 Lucas CR, Korman NJ, Gilliam AC. Granulomatous periorificial dermatitis: a variant of granulomatous rosacea in children? J Cutan Med Surg. 2009;13(2):115-8. 